\title{
ATUAÇÃO MULTIDISCIPLINAR À SAÚDE DO ADOLESCENTE NA ATENÇÃO PRIMÁRIA À SAÚDE
}

\author{
Sebastião Junior Henrique Duarte ${ }^{1}$ \\ Adriana Delmondes de Oliveira ${ }^{2}$ \\ Marina de Souza Morato ${ }^{2}$ \\ Lia Hanna Martins Morita ${ }^{3}$
}

DUARTE, S. J. H.; OLIVEIRA, A. D. de; MORATO, M. de S.; MORITA, L. H. M. Atuação multidisciplinar à saúde do adolescente na atenção primária à saúde. Arq. Ciênc. Saúde UNIPAR, Umuarama, v. 18, n. 1, p. 23-26, jan./mar. 2014

\begin{abstract}
RESUMO: Objetivou-se destacar o conhecimento de profissionais da Estratégia Saúde da Família a respeito de ações desenvolvidas aos adolescentes. Estudo exploratório e descritivo, realizado com 146 profissionais da Estratégia Saúde da Família do município de Cuiabá, MT, a partir de resposta a questionário estruturado contendo dados de caracterização e questões referentes às ações ofertadas aos adolescentes. Os resultados receberam tratamento descritivo e revelaram que a maioria dos participantes era do sexo feminino, a maior frequência do acolhimento e do aconselhamento foi realizado por profissionais do nível médio, enquanto a correspondente às ações educativas são promovidas pelos de nível superior. Concluiu-se que o trabalho multiprofissional é fundamental no cuidado integral aos adolescentes. Recomenda-se que os profissionais reúnam-se periodicamente para o planejamento das atividades a serem realizadas, suprindo as lacunas de conhecimento a respeito das ações ofertadas aos adolescentes.
\end{abstract}

PALAVRAS-CHAVE: Atenção primária à saúde; Saúde do adolescente; Estratégia saúde da família.

\section{ADOLESCENT HEALTH MULTIDISCIPLINARY PRACTICE IN PRIMARY HEALTH CARE}

\begin{abstract}
The objective of this paper was to emphasize the knowledge from professionals in the Family Health Strategy regarding actions taken towards adolescent health. An exploratory and descriptive study among 133 professionals from the family health strategy in Cuiabá, Brazil, was performed based on the answers given to a structured questionnaire with characterization data and issues relating to the actions provided to adolescents. The results were given descriptive treatment and revealed that the majority of participants were female, most often the host and counseling was conducted by professionals from secondary education, while the corresponding education actions are provided by graduated professionals. It was concluded that the multidisciplinary approach is essential for the comprehensive care of adolescents. It is recommended that practitioners get together periodically to plan the activities to be performed, bridging the gaps in knowledge about the actions offered to adolescents.

KEYWORDS: Primary health Care; Adolescent health; Family health strategy.
\end{abstract}

\section{Introdução}

A atenção à saúde do adolescente tem sido um importante desafio tanto para a organização dos serviços de saúde como à sociedade. Nas últimas décadas, a necessidade do estabelecimento de políticas para a adolescência tem-se destacado, considerando o grande contingente populacional que estes grupos representam e, também, pela importância do desenvolvimento integral de suas potencialidades (DUARTE; FERREIRA; SANTOS, 2013).

Uma das políticas de importante destaque é o Programa Saúde do Adolescente (PROSAD), implementado pelo Ministério da Saúde desde o ano de 1989, com o propósito de promover a saúde integral dos adolescentes (BRASIL, 1989).

As ações propostas pelo Programa fundamentam--se, basicamente, na promoção, vigilância, tratamento e reabilitação em saúde, tomando o adolescente como protagonista e estimulando a aquisição de conhecimentos necessários para melhor controle de sua saúde. No PROSAD, a atenção ao adolescente é desenvolvida por equipe multiprofissional, na perspectiva da integralidade do cuidado, levando em consideração as dimensões biopsicossocioespirituais, em constante interação com o meio ambiente e toda a sociedade (BRASIL, 2007).
Ressalta-se que os profissionais atuantes nas equipes da Estratégia Saúde da Família (ESF) devem ser considerados como referência para o primeiro contato da comunidade com os serviços de saúde. No entanto, observa-se que nem todas as equipes da ESF desenvolvem ações do PROSAD.

Partimos do pressuposto de que é possível oferecer atenção integral à saúde do adolescente na perspectiva do PROSAD na medida em que haja ações de sustentabilidade para a efetivação do programa por meio de estrutura de apoio, com equipe multidisciplinar qualificada, tendo como referência uma rede de serviços vinculada a tais ações, e também considerando que os profissionais envolvidos recebam educação permanente com estratégias que favoreçam o diálogo, a troca e a transdisciplinaridade entre os distintos saberes formais e não-formais, de modo a poderem contribuir com as medidas de promoção de saúde em nível individual e coletivo (FERRARI; THOMSON; MELCHIOR, 2006).

Com base nesse pensamento, questionamos: Quais ações são ofertadas aos adolescentes, a partir das necessidades desse grupo populacional? A partir desse interesse, consideramos oportuno, por meio desse estudo empírico, aproximarmo-nos do mundo do trabalho de profissionais envolvidos na implantação do programa, tendo como perspectiva os conceitos de adolescência e de integralidade da atenção à saúde.

DOI: https://doi.org/10.25110/arqsaude.v18i1.2014.5154

${ }^{1}$ Professor Adjunto na Universidade Federal de Mato Grosso do Sul, graduação e mestrado em enfermagem. Líder Grupo de Pesquisa Atenção à Saúde de Indivíduos e Coletividades (ASICO). Endereço para correspondência: UFMS. Av Ranulpho Marques Leal, 3.484, CEP 79620-080, Três Lagoas, MS Telefone (67) 3509-3700

${ }^{2}$ Universidade Federal de Mato Grosso. Acadêmicas de Enfermagem e Estatística, respectivamente. Bolsistas PIBIC.

${ }^{3}$ Professora Assistente na Universidade Federal de Mato Grosso, curso de Estatística. 
O objetivo desse estudo foi destacar o conhecimento de profissionais da Estratégia Saúde da Família a respeito de ações desenvolvidas aos adolescentes.

\section{Material e Método}

Esse estudo exploratório, descritivo, transversal, realizado com Agentes Comunitários de Saúde (ACS), Auxiliares e Técnicos de enfermagem, Enfermeiros e Médicos do município de Cuiabá, MT, no período de setembro de 2011 a julho de 2012, é parte da pesquisa intitulada "Análise da situação de saúde da população cuiabana assistida pelo PETSAÚDE", aprovada pelo Comitê de ética em pesquisas envolvendo seres humanos da Universidade Federal de Mato Grosso, protocolo número 693/2009, subprojetos do Programa Institucional de Bolsas de Iniciação Científica (PIBIC) com o objetivo de avaliar as etapas de estrutura e processo para a implantação do PROSAD em unidades da Estratégia Saúde da Família onde teve implantado o Projeto PETSAÚDE/Saúde da Família em Cuiabá, MT.

Participaram do estudo 146 profissionais, sendo 87 ACS, 24 Auxiliares ou Técnicos de Enfermagem, 18 Enfer- meiros e 17 Médicos, que assinaram o Termo de Consentimento Livre e Esclarecido e aceitaram em contribuir voluntariamente na coleta dos dados, a partir de resposta individual a questionário estruturado, contendo variáveis de caracterização e outras relacionadas a oferta de ações voltadas aos adolescentes.

Pretendeu-se contribuir na (re) estruturação do Programa Saúde do Adolescente em 18 Unidades de Saúde de Cuiabá, MT, na perspectiva do Ministério da Saúde do Brasil.

\section{Resultados}

A maioria dos participantes era do sexo feminino (85\%); com a média de idade de 47 anos (25-60). O relacionamento estável predominou para $46 \%$, no entanto somente $42 \%$ tinham filhos adolescentes, revelando que mais de $40 \%$ dos profissionais possuía contato familiar com adolescentes. Quanto ao tempo de experiência, mais de $50 \%$ possuía menos de um ano de atuação na ESF.

Quanto às ações ofertadas aos adolescentes, a tabela 1 mostra a opinião dos profissionais da ESF a respeito do acolhimento.

Tabela 1: Frequência das respostas de 146 profissionais da Estratégia Saúde da Família de Cuiabá quanto à realização do acolhimento prestado a adolescentes. Cuiabá, 2010.

\begin{tabular}{l|c|c|c|c|c}
\hline \multirow{2}{*}{} & \multicolumn{3}{|c|}{ Acolhimento diário } & \multirow{2}{*}{ Total } \\
\cline { 3 - 6 } \multicolumn{2}{c|}{ Nível de escolaridade } & desconhece & não & sim & \\
& Médio & $16(14,4 \%)$ & $39(35,1 \%)$ & $56(50,5 \%)$ & $111(100 \%)$ \\
& Superior & $4(11,4 \%)$ & $8(22,9 \%)$ & $23(65,7 \%)$ & $35(100 \%)$ \\
\hline \multicolumn{2}{l|}{ Total } & $20(13,7 \%)$ & $47(32,2 \%)$ & $79(54,1 \%)$ & $146(100 \%)$ \\
\hline
\end{tabular}

A tabela 2 ilustra o percentual de respostas referente à disponibilização de dias específicos para o atendimento aos adolescentes no âmbito das unidades de saúde e revela o acesso desse segmento populacional aos profissionais.

Tabela 2: Frequência das respostas de 146 profissionais da Estratégia Saúde da Família de Cuiabá quanto ao atendimento em dias específicos para adolescentes. Cuiabá, 2010.

\begin{tabular}{l|c|c|c|c|c}
\hline \multirow{2}{*}{} & \multicolumn{2}{|c|}{ Atendimento dias específicos adolescentes } & \multirow{2}{*}{ Total } \\
\cline { 3 - 6 } \multicolumn{2}{c|}{ Nível de escolaridade } & desconhece & não & sim & \\
& Médio & $14(12,6 \%)$ & $65(58,6 \%)$ & $32(28,8 \%)$ & $111(100 \%)$ \\
& Superior & $0(, 0 \%)$ & $28(80 \%)$ & $7(20 \%)$ & $35(100 \%)$ \\
\hline \multicolumn{2}{|c|}{ Total } & $14(9,6 \%)$ & $93(63,7 \%)$ & $39(26,7 \%)$ & $146(100 \%)$ \\
\hline
\end{tabular}

O aconselhamento é uma das ações mais realizadas pelos profissionais participantes, conforme descrito na tabela 3.

Tabela 3: Frequência das respostas de 146 profissionais da Estratégia Saúde da Família de Cuiabá quanto ao aconselhamento para adolescentes. Cuiabá, 2010.

\begin{tabular}{l|c|c|c|c|c}
\hline \multirow{2}{*}{} & \multicolumn{3}{|c|}{ Aconselhamento } & \multirow{2}{*}{ Total } \\
\cline { 3 - 6 } \multicolumn{2}{c|}{} & desconhece & não & $\operatorname{sim}$ & \\
\hline \multirow{2}{*}{ Nível de escolaridade } & Médio & $27(24,3 \%)$ & $20(18,0 \%)$ & $64(57,7 \%)$ & $111(100 \%)$ \\
& Superior & $0(, 0 \%)$ & $3(8,6 \%)$ & $32(91,4 \%)$ & $35(100 \%)$ \\
\hline \multicolumn{2}{l|}{ Total } & $27(18,4 \%)$ & $23(15,8 \%)$ & $96(65,8 \%)$ & $146(100 \%)$ \\
\hline
\end{tabular}

Embora no computo geral das atividades educativas não se tenha mais de $50 \%$ de profissionais envolvidos, a tabe- la 4 aponta que os profissionais do nível superior são os que mais promovem essa ação. 
Tabela 4: Frequência das respostas de 146 profissionais da Estratégia Saúde da Família de Cuiabá quanto à realização de ações educativas para adolescentes. Cuiabá, 2010.

\begin{tabular}{l|c|c|c|c|c}
\hline \multicolumn{2}{c|}{} & \multicolumn{2}{|c|}{ Ações educativas adolescentes } & \multirow{2}{*}{ Total } \\
\cline { 3 - 6 } \multicolumn{2}{c|}{} & desconhece & não & sim & \\
\hline \multirow{2}{*}{ Nível de escolaridade } & Médio & $20(18,1 \%)$ & $48(43,2 \%)$ & $43(38,7 \%)$ & $111(100 \%)$ \\
& Superior & $2(5,7 \%)$ & $13(37,2 \%)$ & $20(57,1 \%)$ & $35(100 \%)$ \\
\hline \multicolumn{2}{l|}{ Total } & $22(15,1 \%)$ & $61(41,8 \%)$ & $63(43,1 \%)$ & $146(100 \%)$ \\
\hline
\end{tabular}

Os resultados referentes ao pré-natal da adolescente e seu companheiro são preocupantes, como demonstrados na tabela 5, tendo em vista que mais de $80 \%$ dos participantes não realizam esse tipo de atendimento.

Tabela 5: Frequência das respostas de 146 profissionais da Estratégia Saúde da Família de Cuiabá quanto à realização de pré-natal específico para adolescentes, Cuiabá, 2010.

\begin{tabular}{|c|c|c|c|c|c|}
\hline & & \multicolumn{3}{|c|}{ Pré-natal específico adolescente gestante } & \multirow{2}{*}{ Total } \\
\hline & & desconhece & não & $\operatorname{sim}$ & \\
\hline \multirow{2}{*}{ Nível de escolaridade } & Médio & $14(12,6 \%)$ & $88(79,3 \%)$ & $9(8,1 \%)$ & $111(100 \%)$ \\
\hline & Superior & $0(, 0 \%)$ & $32(91,4 \%)$ & $3(8,6 \%)$ & $35(100 \%)$ \\
\hline \multicolumn{2}{|l|}{ Total } & $14(9,6 \%)$ & $120(82,2 \%)$ & $12(8,2 \%)$ & $146(100 \%)$ \\
\hline
\end{tabular}

Os resultados revelaram que ações assistenciais, como o atendimento pré-natal, não fazem parte das rotinas da maioria dos profissionais participantes. Tal situação compromete $o$ atendimento integral.

\section{Discussão}

O percentual de mulheres encontrada entre as participantes assemelha-se a outros estudos onde predominaram as mulheres como a maioria entre os profissionais da área da saúde, com destaque à equipe de enfermagem e os Agentes Comunitários de Saúde. Tal fato é considerado como relevante no trabalho com adolescentes, especialmente quando a profissional possui experiência familiar nessa etapa do ciclo de vida (DUARTE; MAMEDE, 2012).

Quanto à realização do acolhimento chama atenção que mais de $40 \%$ dos participantes não realizam ou desconhecem essa tecnologia que promove a atenção à saúde. Ressalta-se que o acolhimento é considerado como uma das estratégias para garantir a efetivação do Sistema Único de Saúde, conforme estabelecido na Constituição Federal de 88 e na Lei 8080/90, seguindo os princípios da universalidade do acesso, integralidade das ações, equidade, qualidade e responsabilidade. $\mathrm{O}$ acolhimento favorece a humanização nas relações entre profissionais e usuários. Assim, por meio do acolhimento, é possível que os adolescentes sejam ouvidos com atenção, recebam informações e atendimento satisfatório (OLIVEIRA et al., 2009).

Outra informação relevante refere-se à agenda dos profissionais, em que a maioria dos respondentes afirmou não estipular dia da semana para o atendimento aos adolescentes. Por um lado, se a demanda for espontânea, ou seja, acesso irrestrito dos adolescentes aos profissionais de saúde quando esses buscarem os serviços de saúde, então o encontrado propicia o atendimento integral, do contrário, é preciso (re) organização das agendas, incluindo a atenção aos adolescentes, como ocorre em outros grupos populacionais, por exemplo, crianças e mulheres.

Com relação ao aconselhamento, os resultados evi- denciaram que os adolescentes são assistidos com esse método do cuidar, favorecendo a prevenção de doenças e agravos, bem como a manutenção de hábitos saudáveis. Vale destacar que o aconselhamento pode se dar em ações educativas com grupos de adolescentes, visto que cabe ao profissional desenvolver ações educativas a partir das necessidades identificadas pelo próprio grupo, considerando o contexto histórico, político, econômico e sociocultural da região (OLIVEIRA et al., 2009).

Em relação à ações educativas nos chamou atenção, o número de profissionais do nível médio que desconhecem se há ações educativas para adolescentes nas equipes em que atuam. A situação consiste em alerta, visto que o trabalho do Agente Comunitário de Saúde é eminentemente voltado para a educação em saúde. Contudo, a maioria dos profissionais do nível superior relatou o envolvimento nas atividades educativas. Com isso, destacamos a importância do planejamento das ações a serem desenvolvidas pela equipe, que deverão ser tratadas em reunião, visando a autonomia dos adolescentes quanto ao estilo de vida a partir de mudanças pela educação (FEIRE, 2001).

Os resultados referentes ao pré-natal para adolescentes evidenciaram o quanto os profissionais participantes carecem de inserir esse tipo de atendimento à clientela, visto que a intenção é o atendimento integral do adolescente. Embora a gravidez na adolescência seja preocupante, é preciso o envolvimento dos profissionais da saúde, seja na fase de esclarecimento da saúde sexual e reprodutiva, na divulgação de métodos contraceptivos disponível no serviço público, como na realização do pré-natal.

Segundo o Ministério da Saúde (2006), o principal objetivo da atenção pré-natal e puerperal é acolher a mulher desde o início da gravidez, assegurando, ao fim da gestação, o nascimento de uma criança saudável e a garantia do bem-estar materno e neonatal.

Uma atenção pré-natal e puerperal qualificada e humanizada se dá por meio de incorporação de condutas acolhedoras e sem intervenções desnecessárias; do fácil acesso aos serviços de saúde de qualidade, com ações que integrem 
todos os níveis da atenção: promoção, prevenção e assistência à saúde da gestante e do recém-nascido, desde o atendimento ambulatorial básico ao atendimento hospitalar para alto risco (PENNA et al., 2012).

Trabalhar educação para saúde dos adolescentes difere da assistência clínica individual e da simples informação. O modelo a ser desenvolvido deve permitir discussão sobre as razões de adoção de comportamento preventivo, e o desenvolvimento de habilidades que permitam a resistência às pressões externas, a expressão de sentimentos, opiniões, dúvidas, inseguranças, medos e preconceitos, de forma a dar condições para o enfrentamento e a resolução de problemas e dificuldades do dia a dia (SAMPAIO et al., 2010).

Os resultados encontrados nesse estudo mostram que os adolescentes estão sendo assistidos, mesmo sem a inclusão desse segmento populacional nas atividades do cotidiano em todas as equipes, constituindo em motivação para a (re) organização da atenção à saúde do adolescente, na perspectiva da integralidade do cuidado.

\section{Conclusão}

O estudo permitiu destacar a opinião de profissionais atuantes em equipes da Estratégia Saúde da Família quanto as ações desenvolvidas no âmbito do Programa Saúde do Adolescente, permitindo concluir que há acesso aos profissionais de saúde, no entanto algumas lacunas carecem ser discutidas e melhoradas, com enfoque às atividades educativas e a realização do pré-natal da adolescente.

É preciso que o processo de trabalho em saúde do adolescente leve em consideração as atividades propostas pelo Programa Saúde do Adolescente, a serem desenvolvidas por equipe multiprofissional, em busca da promoção da saúde por meio da informação que permita a autonomia do autocuidado, bem como o favorecimento na mudança do estilo de vida desfavorável à saúde, ainda, o estímulo à participação dos adolescentes nos serviços de saúde.

A necessidade da existência de serviços de saúde de qualidade tem sido colocada como um desafio para o alcance de melhores condições de saúde dos adolescentes, o que significa compreender a importância das dimensões econômica, social e cultural que permeiam a vida desses grupos.

\section{Referências}

BRASIL. Ministério da Saúde. Pré Natal e Puerpério; Atenção Qualificada e Humanizada. Série A. Normas e Manuais Técnicos. Brasília: DF. 2006.

BRASIL. Ministério da Saúde. Saúde Integral de Adolescentes e Jovens; Orientações para a Organização de Serviços de Saúde. Série A. Normas e Manuais Técnicos. Brasília: DF. 2007.

. Secretária Nacional de Programas Especiais de Saúde, Divisão Nacional de DST/ AIDS. Programa Nacional de Integração Integral ao Adolescente. Brasília: Ministério da Saúde, 1989.

DUARTE, S. J. H. D.; FERREIRA, S. F.; SANTOS, N.

C. Desafio de enfermeiros da Estratégia Saúde da Família na implantação do Programa Saúde do Adolescente. Rev. Eletr. Enf. Goiania, v. 15, n. 2, p. 479-486, jun. 2013.

DUARTE, S. J. H.D.; MAMEDE, M. V. Estudo das competências essenciais na atenção pré-natal: ações da equipe de enfermagem em Cuiabá, MT. Enfermagem em foco, v. 3, n. 2, p. 75-80, 2012.

FREIRE, P. Educação e mudança. 24. ed. Rio Janeiro: Paz e Terra, 2001.

FERRARI, R. A. P.; THOMSON, Z.; MELCHIOR, R. Atenção à saúde dos adolescentes: percepção dos médicos e enfermeiros das equipes da saúde da família. Cad. Saúde Pública, v. 22, n. 11, p. 2491-2495, 2006.

OLIVEIRA, C. D. et al. Conhecimentos e práticas de adolescentes acerca das DST/HIV/AIDS em duas escolas públicas municipais do Rio de Janeiro, Esc. Anna Nery Rev. de Enfermagem, v. 13, n. 4, p. 833-841, 2009.

PENNA, L. H. G. et al. A maternidade no contexto de abrigamento: concepções das adolescentes abrigadas. Rev. esc. enferm. USP, São Paulo, v. 46, n. 3, p. 544-548, 2012.

SAMPAIO, J. et al. Promoção da saúde sexual: desafios no Vale do São Francisco. Psicol. Soc. v. 22, n. 3, p. 499-506, 2010 . 